\title{
Neurotropismo de SARS-CoV-2: Possíveis impactos da COVID-19 em portadores de
}

\section{Esclerose Lateral Amiotrófica}

\author{
Neurotropism of SARS-CoV-2: Possible impacts of COVID-19 in patients with Amyotrophic \\ Lateral Sclerosis \\ Neurotropismo del SARS-CoV-2: Posibles impactos del COVID-19 en pacientes con Esclerosis \\ Lateral Amiotrófica
}

Recebido: 24/05/2021 | Revisado: 01/06/2021 | Aceito: 08/06/2021 | Publicado: 21/06/2021

\author{
Nayane Soares de Lima \\ ORCID: https://orcid.org/0000-0002-5585-6768 \\ Universidade Federal de Goiás, Brasil \\ E-mail: nayanes00@gmail.com \\ Caroline Christine Pincela da Costa \\ ORCID: https://orcid.org/0000-0002-6732-913X \\ Universidade Federal de Goiás, Brasil \\ E-mail: carolinepincela@gmail.com \\ Angela Adamski da Silva Reis \\ ORCID: https://orcid.org/0000-0002-8281-7334 \\ Universidade Federal de Goiás, Brasil \\ E-mail: angela@ufg.br \\ Rodrigo da Silva Santos \\ ORCID: https://orcid.org/0000-0002-9480-4362 \\ Universidade Federal de Goiás, Brasil \\ E-mail: rdssantos@ufg.br
}

\begin{abstract}
Resumo
A esclerose lateral amiotrófica é uma doença neuromuscular degenerativa que acomete os neurônios motores superiores e inferiores. O estado de debilitação motora e funcional decorrente da progressão da doença os expõe a um risco possivelmente fatal no caso de infeção pelo SARS-CoV-2, especialmente devido ao severo acometimento pulmonar, característico do avanço da patologia degenerativa em questão. Nesta revisão, elencamos os principais mecanismos associativos entre a doença e a COVID-19, destacando, principalmente, os aspectos virais neuroinvasivos. $\mathrm{O}$ estudo trata-se de uma pesquisa qualitativa descritiva, realizada mediante revisão narrativa da literatura. A literatura acerca do novo coronavírus tem demonstrado a capacidade viral de neuroinvasão e neurotrópica do SARS-CoV-2. Atualmente, as evidências comprovam que a infecção não é restrita ao trato respiratório, o qual é o sítio primário da infecção. Com base nos achados, propõe-se a existência de três cenários viáveis no impacto do SARS-CoV-2 e a neuroinvasão: (1) indução, de forma direta, às alterações neurológicas; (2) agravamento de condições neurológicas previamente instaladas; e (3) aumento na susceptibilidade ou da deterioração de danos provocados por outras injúrias, nesse caso, considerando os portadores de ELA. Conclui-se que existe a possibilidade de um período latente do SARS-CoV-2 no SNC. Dessa forma, é crucial a observação de sintomas neurológicos posteriores no individuo, decorrentes da reativação viral, resultando em progressão continua dos processos neuroinflamatórios associados à ELA. As capacidades neuroinvasivas e neurotrópicas do vírus também devem ser observadas em longo prazo para elucidar se as doenças neurodegenerativas, como a ELA conferem vulnerabilidade direta a COVID-19.
\end{abstract}

Palavras-chave: Alterações neurológicas; Doenças neuromusculares; Esclerose lateral amiotrófica; SARS-CoV-2; Inflamação.

\begin{abstract}
Amyotrophic lateral sclerosis is a degenerative neuromuscular disease that affects the upper and lower motor neurons. The state of motor and functional impairment resulting from the progression of the disease exposes them to a possibly fatal risk in the case of infection by SARS-CoV-2, especially due to the severe pulmonary involvement, characteristic of the advancement of the degenerative pathology in question. In this review, we list the main associative mechanisms between the disease and COVID-19, highlighting, mainly, the neuroinvasive viral aspects. The study is a qualitative descriptive research, carried out through a narrative review of the literature. The literature on the new coronavirus has demonstrated the viral neuroinvasion and neurotropic capacity of SARS-CoV-2. Currently, the evidence proves that infection is not restricted to the respiratory tract, which is the primary site of the infection. Based on the findings, it is
\end{abstract}


proposed that there are three viable scenarios on the impact of SARS-CoV-2 and neuroinvasion: (1) direct induction of neurological changes; (2) worsening of previously installed neurological conditions; and (3) increased susceptibility or deterioration of damage caused by other injuries, in this case, considering ALS patients. It is concluded that there is a possibility of a latent period of SARS-CoV-2 in the CNS. Thus, it is crucial to observe subsequent neurological symptoms in the individual, resulting from viral reactivation, resulting in continuous progression of the neuroinflammatory processes associated with ALS. The neuroinvasive and neurotropic capacities of the virus must also be observed in the long term to elucidate whether neurodegenerative diseases, such as ALS, confer direct vulnerability to COVID-19.

Keywords: Neurological alterations; Neuromuscular diseases; Amyotrophic lateral sclerosis; SARS-CoV-2; Inflammation.

\section{Resumen}

La esclerosis lateral amiotrófica es una enfermedad neuromuscular degenerativa que afecta a las neuronas motoras superiores e inferiores. El estado de debilitamiento motor y funcional resultante de la progresión de la enfermedad les expone a un riesgo posiblemente mortal en caso de infección por el SARS-CoV-2, especialmente debido a la grave afectación pulmonar, característica de la progresión de la patología degenerativa en cuestión. En esta revisión, enumeramos los principales mecanismos de asociación entre la enfermedad y la COVID-19, destacando principalmente los aspectos virales neuroinvasivos. El estudio se basa en una investigación cualitativa descriptiva, realizada mediante una revisión narrativa de la literatura. La literatura sobre el nuevo coronavirus ha demostrado la capacidad viral de neuroinvasión y neurotrópica del SARS-CoV-2. Actualmente, la evidencia prueba que la infección no se limita al tracto respiratorio, que es el sitio principal de la infección. Basándonos en los hallazgos, proponemos la existencia de tres escenarios viables en el impacto del SARS-CoV-2 y la neuroinvasión: (1) inducción, de forma directa, a alteraciones neurológicas; (2) empeoramiento de condiciones neurológicas previamente instaladas; y (3) aumento de la susceptibilidad o del deterioro de daños causados por otras lesiones, en este caso, considerando a los portadores de ELA. Concluimos que existe la posibilidad de un periodo de latencia del SARS-CoV-2 en el SNC. Por lo tanto, es crucial observar los síntomas neurológicos subsiguientes en el individuo, resultantes de la reactivación viral, lo que resulta en la progresión continua de los procesos neuroinflamatorios asociados con la ELA. La capacidad neuroinvasiva y neurotrópica del virus también debería observarse a largo plazo para dilucidar si las enfermedades neurodegenerativas como la ELA confieren una vulnerabilidad directa a COVID-19.

Palabras clave: Cambios neurológicos; Enfermedades neuromusculares; Esclerosis lateral amiotrófica; SARS-CoV2; Inflamación.

\section{Introdução}

\subsection{Esclerose Lateral Amiotrófica}

A Esclerose Lateral Amiotrófica (ELA) é uma doença neurodegenerativa caracterizada pela degeneração progressiva de neurônios motores superiores e inferiores no cérebro e medula espinhal, acarretando em paralisia e atrofia muscular (Chia et al., 2018). Por também afetar músculos como o diafragma, a falência respiratória é a principal causa de óbito destes pacientes, ocorrendo entre dois a cinco anos após o início dos primeiros sintomas (Hardiman et al., 2017; van Es et al., 2017).

O fator preditivo para o desenvolvimento da ELA é a idade, acometendo principalmente indivíduos entre 55 e 75 anos (Chia et al., 2018). A incidência anual da doença varia mundialmente entre 1,0-1,7 casos a cada 100.000 habitantes, enquanto sua prevalência é de 5,0 casos por 100.000 habitantes (Oskarsson et al., 2018).

A ELA possui diferentes fenótipos e os sintomas podem variar entre pessoas de acordo com o local inicialmente afetado. Na ELA clássica, os sintomas são predominantemente dos membros inferiores e incluem disfunção motora, fraqueza muscular, cãibras e espasticidade. Quando acometido pela forma bulbar, o paciente demonstra sintomas iniciais como disartria, disfagia, disfonia e declínio da função respiratória, referentes à degeneração dos neurônios motores do tronco cerebral (van Es et al., 2017; Walhout et al., 2018).

A etiologia da doença permanece desconhecida. Contudo, diversos mecanismos patológicos têm sido relatados, como disfunção mitocondrial e estresse oxidativo, falha no reparo do DNA, distúrbios no metabolismo do RNA, agregação proteica, excitotoxicidade do glutamato, mutações genéticas e neuroinflamação (Mejzini et al., 2019; Taylor et al., 2016). Os fatores genéticos parecem desempenhar um papel importante no desenvolvimento da doença. Algumas variantes polimórficas em genes como SOD1, C9orf72, FUS, VAPB e TARDBP são relacionadas tanto com a forma esporádica da doença, que ocorre em 
95\% dos casos de ELA e não possuem causa aparente, quanto na forma familiar, determinada por casos previamente relatados na família, correspondendo à 5\% dos casos da patologia (Ajroud-Driss \& Siddique, 2015; Chia et al., 2018).

A atual pandemia originada pelo SARS-CoV-2 é um fator preocupante não só para a população em geral, mas principalmente para pessoas dos grupos de risco, segundo a Organização Mundial de Saúde (OMS). Como uma doença rara e com background genético, pacientes portadores de ELA constituem um grupo altamente vulnerável à infecção pelo vírus (Guidon \& Amato, 2020). Além disso, a idade avançada ao qual a ELA normalmente acomete os indivíduos, somado ao declínio da capacidade motora e comprometimento respiratório, causados pela progressão da doença, são agravantes para a COVID-19, doença causada pelo novo coronavírus (Hardiman et al., 2017). Adicionalmente, os portadores de ELA recorrentemente necessitam de apoio ou cuidadores para movimentar-se, o que eleva o risco de contaminação comunitária ou por toques em superfícies contaminadas, tornando o cenário elevadamente preocupante (Oh \& Kim, 2017).

Assim, a vacinação deve ser prioritária a grupos, com doenças raras, como a ELA, uma vez que a condição progressivamente deteriorada desses pacientes podem favorecer desfechos negativos em caso de infecção pelo vírus. Ademais, o programa brasileiro de imunização não estipulou estratos para a vacinação desse grupo, bem como de seus cuidadores. Esses fatores somam preocupações quanto ao possível risco fatal do vírus nesses pacientes.

\subsection{SARS-CoV-2 e COVID-19}

Patógenos reemergentes são um desafio preocupante para a saúde pública. Os coronavírus são vírus envelopados de RNA com polaridade positiva, pertencente à família Coronaviridae. Esse grupo viral é composto por quatro gêneros: alfa, beta, delta e gammacoronavírus, sendo alfa e beta os gêneros mais recorrentes em infecções humanas. São amplamente distribuídos entre mamíferos e conhecidos por causarem, principalmente, doenças respiratórias, porém também estão patologicamente associados a alterações hepáticas, entéricas e neurológicas (Zhu et al., 2020).

O SARS-CoV-2, sigla para Síndrome Respiratória Aguda Grave 2, é um betacoronavírus, patógeno responsável pela doença do coronavírus 19 (COVID-19). Outras duas cepas semelhantes, altamente patogênicas, também causaram graves surtos, como a SARS-CoV entre 2002/2003, no qual o SARS-CoV-2 compartilha alta homologia em sua sequência nucleotídica, e em 2012, com o coronavírus da síndrome respiratória do Oriente Médio (MERS-CoV) (Wilson \& Jack, 2020; Zhu et al., 2020).

A alta prevalência e distribuição dos coronavírus implicam em uma ampla diversidade genética e recombinação frequente de seus genomas, o que explica a heterogeneidade intra e interespécies. Devido a isso, a relação patógeno-hospedeiro é aperfeiçoada objetivando a propagação da espécie, e assim, o surgimento periódico de novos coronavírus é uma possibilidade (Carod Artal, 2020; Zhu et al., 2020).

O SARS-Cov-2 é o sétimo coronavírus a causar doenças em humanos (Lippi et al., 2020) e o terceiro, desde 2002 a ser transmitido de animais para humanos, acarretando em patologias respiratórias, como pneumonias. Sua origem tem sido estudada, entretanto, propõe-se uma fonte animal para transmissão primária do vírus (Filatov et al., 2020). As pneumonias atípicas decorrentes desta infecção viral, denominada de COVID-19, surgiram em dezembro de 2019 na cidade de Wuhan na China, espalhando-se pelo mundo, ocasionando a atual pandemia. O termo coronavírus foi designado devido à aparência em forma de coroa ao qual a estrutura viral se assemelha (Yang et al., 2020).

Os sintomas da COVID-19 podem incluir febre, tosse, perda de olfato e paladar, dor de garganta, dor nas pernas, dor de cabeça, diarreia e fadiga. Os sintomas parecem variar entre indivíduos (Filatov et al., 2020), mas a maioria dos pacientes infectados com SARS-CoV-2 é assintomática ou desenvolvem sintomas leves a moderados, não necessitando de intervenções hospitalares (De Felice et al., 2020). Todavia, alguns podem desenvolver desde pneumonia grave com insuficiência respiratória à choque séptico podendo evoluir ao óbito (Filatov et al., 2020). 
Os dados epidemiológicos e clínicos da doença são limitados e constantemente atualizados, contudo, sugerem diferenças na transmissão da doença em relação ao SARS-CoV (Gorbalenya et al., 2020). Diversas pesquisas estão em andamento para melhor compreender os mecanismos de transmissão viral, todavia, a principal via de contaminação parece ser interpessoal, por meio de gotículas de saliva expelidas pela boca ou nariz, seja por tosse ou espirros, assemelhando-se a transmissão do vírus influenza. Além disso, estudos mostram uma atividade viral persistente sob diferentes superfícies, podendo estar viável para a contaminação por longos períodos de horas, demonstrando assim, ser um importante meio de transmissão (van Doremalen et al., 2020).

A relação vírus-hospedeiro é mediada pelo reconhecimento da enzima conversora de angiotensina 2 (ECA2) através da proteína Spike (S), componente do envelope viral. A enzima ECA2 é amplamente expressa em diversos órgãos como no trato gastrointestinal e urinário, rins, mucosa nasal, epitélio das vias aéreas (incluindo parênquima pulmonar), tecidos linfóides, órgãos reprodutivos, endotélio vascular e cérebro (M.-Y. Li et al., 2020). A infecção pelo SARS-CoV-2 é mais intensa em portadores de doenças crônicas, como a ELA. Sendo assim, tais patologias conferem susceptibilidade maior a formas graves da COVID-19 (M.-Y. Li et al., 2020; Y. Li et al., 2020).

Dessa forma considerando o exposto acima, nosso estudo tem por objetivo reunir informações acerca da potencial ação neuroinvasiva proporcionada pelo SARS-CoV-2, diante da possibilidade deste, ocasionar prejuízos para a saúde dos portadores da ELA, bem como acarretar em um desfecho na qual existe a possibilidade de aceleração do óbito de indivíduos.

\section{Metodologia}

O presente estudo trata-se de uma pesquisa qualitativa descritiva, realizada mediante uma revisão narrativa da literatura. O processo realizado através da revisão narrativa permite ao pesquisador a inclusão de diversos estudos recentes acerca de uma temática de interesse, os quais podem conter abordagens metodológicas distintas o que por sua vez, contribui com diferentes visões de uma mesma problemática, favorecendo o processo do conhecimento (Crepaldi et al., 2020; Estrela, 2018).

Para o desenvolvimento do estudo foram considerados artigos escritos em português e inglês, os quais publicados entre 2015 e 2020. Foram considerados os seguintes bancos de dados: Scientific Eletronic Library Online (SciELO), National Library of Medicine (PubMed/NCBI) e Biblioteca Virtual em Saúde (BVS). A busca ocorreu durante o mês de dezembro de 2020 a partir do uso de descritores e palavras-chave como "Coronavirus"; "SARS-CoV"; "Pandemic"; "COVID-19"; "Neurotropism"; "Amyotrophic Lateral Sclerosis"; "Motor Neuron Disease"; "Neurological alterations"; "Neurological complications", aplicando os operadores booleanos "AND" e/ou "OR" para refinar as buscas, essas foram realizadas nos idiomas inglês e português para abranger o maior número possível de publicações. Após análise bibliográfica, e seleção através da leitura de títulos e resumos, os estudos com temática de interesse foram inclusos na escrita da revisão.

\section{Resultados e Discussão}

\subsection{ELA e COVID-19: Comprometimento pulmonar}

O SARS-CoV-2 consegue replicar-se e proliferar-se em pneumócitos, um tipo de célula alveolar, acarretando em um estado inflamatório intersticial e alveolar difuso, através da produção de exsudato. Em casos mais graves, pode ocorrer a formação de membranas, prejudicando as trocas gasosas nos alvéolos pulmonares (Carod Artal, 2020). Considerando a ampla expressão de ECA2 nas células pulmonares, a concentração viral aumentada neste sítio anatômico contribui para um estado hipóxico grave (M.-Y. Li et al., 2020).

Pacientes com o sistema respiratório afetado evoluem para quadros graves de COVID-19 e demandam internações em unidades de terapia intensiva (UTIs). Assim, necessitam de intervenções respiratórias em procedimentos invasivos ou não- 
invasivos, como ventilação mecânica ou oxigenoterapia, uma vez que não conseguem respirar espontaneamente (Bohmwald et al., 2018).

Adicionalmente, o estado hipóxico decorrente de quadros graves da infecção influencia na instalação de um metabolismo anaeróbico no SNC, causando edema celular e intersticial, além de isquemia e vasodilatação na circulação cerebral. Mediante essas circunstâncias, episódios de síncope, crises anóxicas e acidentes vasculares cerebrais podem acontecer (Carod Artal, 2020).

Com a progressão clínica da ELA, os portadores da doença desenvolvem um declínio na função respiratória, culminando em paralisia diafragmática, a principal causa de morte deste grupo (Hardiman et al., 2017; van Es et al., 2017). Assumindo o comprometimento respiratório como a principal complicação da infecção pelo SARS-CoV-2, que pode ser mais grave nestes pacientes por serem do grupo de risco, o acometimento simultâneo destas patologias pode resultar na aceleração de um desfecho fatal (Guidon \& Amato, 2020).

\subsection{Comprometimento neurológico}

Um importante agravante da COVID-19 pode ser o comprometimento do sistema nervoso central (SNC). Os efeitos neurodegenerativos à longo prazo induzidos por SARS-CoV-2 ainda não estão claros, mas fundamenta-se nas experiências e relatos com outros coronavírus, como SARS-CoV e MERS-CoV, bem como outros vírus respiratórios, com o da influenza, zika vírus e Epstein Barr (M.-Y. Li et al., 2020; Meinhardt et al., 2021; Wilson \& Jack, 2020).

Para alguns vírus respiratórios, como o vírus sincicial (VSR), complicações no SNC como encefalites, crises epilépticas, cerebelite e ataxia são relatadas. Outros vírus gripais também estão relacionados com o desenvolvimento de meningite, encefalopatia necrosante e síndrome de Guillain-Barré, uma patologia neuromuscular (Bohmwald et al., 2018; Guidon \& Amato, 2020).

Um número crescente de estudos com coronavírus têm demonstrado a capacidade viral de neuroinvasão, induzindo a neuropatologias (neurovirulência), assim, não estando restritos ao trato respiratório, o qual é o sítio primário da infecção (Carod Artal, 2020). A neuro-deposição viral foi demonstrada em diversos betacoronavírus, como SARS-CoV, MERS-CoV, hCoV-229E, hCoV-OC43, NL63, HKU1; (M.-Y. Li et al., 2020; Y. Li et al., 2020). Cepas 229E, OC43 e 293, por exemplo, já foram identificadas em pacientes com esclerose múltipla (Wilson \& Jack, 2020).

Ademais, a infecção causada por SARS-CoV, vírus anterior, leva ao desenvolvimento de encefalites com quadros convulsivos epiléticos e polineuropatias. Já o acometimento pelo MERS-CoV está associado a episódios de alteração da consciência, variando de confusão mental ao coma, e também quadros de ataxia e déficit motor (Bohmwald et al., 2018).

Atualmente, relatos na literatura destacam uma diversidade de eventos neurológicos associados à infecção pelo SARS-CoV-2. Revisões sistemáticas descrevem dores de cabeça, consciência alterada, tontura, e crises epilépticas estão entre os principais sintomas neurológicos de pacientes diagnosticados com COVID-19 (Correia et al., 2020; Montalvan et al., 2020). Derrames e hemorragias também tem sido recorrentemente citados (Zhou et al., 2020).

As características de neuroinvasão e neurovirulência embasam-se nas evidências de: (1) infiltração do SNC por outros vírus respiratórios, (2) danos neurológicos causados por outras espécies de coronavírus e (3) pacientes com COVID-19 que apresentaram manifestações neurológicas, como tontura, dor de cabeça, perda do olfato e/ou paladar, náuseas e êmeses (Carod Artal, 2020; Y. Li et al., 2020).

O mecanismo de ação para a invasão neurológica viral ainda não está claro. Postula-se que uma das principais vias de proliferação do vírus ocorra por meio de terminais nervosos periféricos, podendo atingir o SNC através dos nervos olfativos ou vias sinápticas, podendo estar associado à alta expressão de ECA2 nas células da mucosa nasal (Das et al., 2020). No entanto, 
mecanismos como vias de circulação sanguínea, lesões por hipóxia e síndrome da tempestade de citocinas também são relatadas (Wilson \& Jack, 2020).

Estudos experimentais com cepas SARS-CoV relataram intensa infecção viral no cérebro de cobaias, sendo o tronco encefálico a região mais prejudicada. Todavia, existe também a possibilidade do vírus afetar o córtex cerebral e o hipotálamo (Yashavantha Rao \& Jayabaskaran, 2020). A possibilidade de acometimento dos neurônios da região bulbar também é potencialmente grave. Por regularem funções respiratórias, pulmonares e cardíacas, danos nestes neurônios podem resultar em desconforto respiratório crônico com consequências irreversíveis, evoluindo para o óbito do paciente (Bohmwald et al., 2018; Das et al., 2020).

Apesar do neurotropismo conhecido dos coronavírus anteriores, as complicações neurológicas causadas pela infecção do SARS-CoV-2 ainda não estão bem elucidadas. Contudo, alguns estudos de necropsia relataram a presença de edemas cerebrais e neurodegeneração em pacientes que vieram a óbito pela COVID-19. É sabido que pacientes com infecções graves possuem uma maior predisposição ao desenvolvimento de sintomas neurológicos comparados aos com infecções brandas. Adicionalmente, a preexistência de doenças neurológicas confere risco maior de neurotropismo quando infectados pelo SARSCOV-2 (Das et al., 2020; Yashavantha Rao \& Jayabaskaran, 2020).

Propõe-se a existência de três cenários viáveis no impacto do SARS-CoV-2 e a neuroinvasão: (1) indução, de forma direta, às alterações neurológicas; (2) agravamento de condições neurológicas previamente instaladas; e (3) aumento na susceptibilidade ou da deterioração de danos provocados por outras injúrias (De Felice et al., 2020).

\subsection{SARS-CoV-2 e células gliais}

A neuroinvasão causada por vírus respiratórios pode afetar tanto neurônios, quanto células gliais, característica denominada neurotropismo. O dano neurológico causado pelo SARS-CoV-2 é, adicionalmente, imunomediado. O novo coronavírus possui a capacidade de infectar macrófagos, astrócitos e micróglia (Carod Artal, 2020).

Uma das rotas propostas para a invasão do vírus ao SNC é a disseminação hematógena. Por essa via de entrada, o vírus pode infectar células endoteliais da barreira hematoencefálica (BHE), possivelmente ligando-se aos receptores ECA2, ou mesmo utilizar de células leucocitárias para disseminar-se. Nesse cenário, o vírus pode infectar monócitos e macrófagos, que por sua vez ultrapassam a BHE e induzem a ativação microglial (Zhou et al., 2020).

A micróglia, célula imune do SNC, pode ser ativada tanto por mecanismos endógenos, como a própria neurodegeneração na ELA, quanto exógenos, no caso de SARS-CoV-2. A infecção viral induz a ativação destas células, que consequentemente liberam mediadores pró-inflamatórios, como as interleucinas (IL) 6, IL-12, IL-15 e fator de necrose tumoral alfa (TNF- $\alpha$ ) (Carod Artal, 2020; Toljan, 2020).

A ativação da micróglia concomitante ao processo de neuroinflamação instalado pela ELA pode induzir a formação de uma síndrome hiperinflamatória, denominada tempestade de citocinas (Carod Artal, 2020). Este cenário também foi associado a outras comorbidades, como em portadores de diabetes mellitus que desenvolveram a COVID-19, gerando um quadro de falência múltipla de órgãos (Prado et al., 2018).

Os mecanismos imunológicos envolvidos na tempestade de citocinas estão frequentemente implicados na patogênese e progressão de diversas doenças neurodegenerativas. Na ELA, a tempestade de citocinas pode acentuar o processo neuroinflamatório e acelerar a degeneração das células neuromotoras (Mehra et al., 2020; Serrano-Castro et al., 2020; Toljan, 2020).

Além disso, por transpor a BHE, previamente lesionada pelo processo neurodegenerativo na ELA, o SARS-CoV-2 pode acentuar o processo de recrutamento de células imunes periféricas bem como mediadores inflamatórios sistêmicos, contribuindo com o processo neuroinflamatório e conferindo susceptibilidade aumentada a síndromes neurológicas (De Felice 
et al., 2020; Mehra et al., 2020). Além disso, também torna os pacientes mais susceptíveis a eventos cerebrovasculares e formação de trombos (Zhou et al., 2020).

Adicionalmente, o sistema imune inato é a primeira linha de defesa humana contra patógenos. Por ser uma nova cepa, a memória imunológica para este antígeno é inexistente. Esse quadro induz a uma resposta imunológica intensa e desregulada, e, além de poder explicar a magnitude dos sintomas associados à infecção, também pode atuar como um cofator na aceleração da neurodegeneração (De Felice et al., 2020; Mehra et al., 2020).

Até o presente momento, ainda não existe um tratamento precoce e efetivo para a COVID-19, baseado em evidências científicas. Contrariamente, alguns fármacos, como a cloroquina e a hidroxicloroquina (Mehra et al., 2020; Tipton \& Wszolek, 2020) estão associados a um potencial efeito colateral neuromuscular (M.-Y. Li et al., 2020; Y. Li et al., 2020), o que pode intensificar os sintomas da ELA. Por serem altamente vulneráveis a uma infecção possivelmente fatal, a adoção de medidas cautelosas de prevenção tornam-se cruciais (Guidon \& Amato, 2020). A inclusão desse grupo nos estratos prioritários da vacinação também é uma cláusula emergente e de demasiada preocupação, visto as atuais complicações neurológicas provocadas pelo SARS-CoV-2. Tanto a COVID-19 quanto a ELA comprometem além do sistema respiratórios e a coexistência das duas patologias pode ser fatal para os portadores de ELA.

\section{Conclusão}

Muito do conhecimento sobre o SARS-CoV-2 é originário de estudos precedentes de outros coronavírus humanos, como o SARS-CoV e o MERS-CoV (Tipton \& Wszolek, 2020). A COVID-19 é um estímulo para a intensificação/progressão da doença em diversos distúrbios neuromusculares, hereditários e imunomediados (Guidon \& Amato, 2020). Contudo ainda não há dados sobre a seriedade deste risco para tais distúrbios, especialmente nos casos de doenças raras, como a ELA.

Aspectos como o grau do déficit respiratório, acometimento bulbar, comorbidades e fisiopatologia subjacente a ELA devem ser considerados no estudo do impacto da infecção viral nestas doenças (Guidon \& Amato, 2020). Além disso, sabe-se que condições virais, como a taxa de mutagenicidade do vírus, e fatores associados ao hospedeiro, como a idade avançada, imunossupressão, comorbidades pré-existentes podem influenciar nos diferentes níveis de neurotropismo, neurovirulência e neuroinvasão da SARS-CoV-2 em humanos (Carod Artal, 2020).

Estudos sobre as complicações e associações do SARS-CoV com doenças neuromusculares/neurodegenerativas são escassos. Diversas lacunas estão abertas, referentes, principalmente a impactos relacionados direta ou indiretamente à COVID19. Além disso, os efeitos deletérios decorrentes da infecção por SARS-CoV-2 não se limitam aos neuromusculares, mas a todas as áreas neurológicas (M.-Y. Li et al., 2020).

Adicionalmente, a possibilidade de o SARS-CoV-2 permanecer latente no SNC não deve ser descartada. Neste cenário, é crucial a observação de sintomas neurológicos tardios, decorrentes da reativação viral, resultando na reativação dos processos neuroinflamatórios associados a ELA (Mehra et al., 2020; Serrano-Castro et al., 2020). As capacidades neuroinvasivas e neurotrópicas do SARS-CoV-2 também devem ser observadas em longo prazo para elucidar se as doenças neurodegenerativas, como Parkinson e a ELA conferem vulnerabilidade direta a COVID-19 (Tipton \& Wszolek, 2020).

Dessa forma, destaca-se a importância de estudos adicionais para melhor compreender os mecanismos subjacentes à interação entre o Sars-cov-2 e as doenças neurodegenerativas em especial a ELA. A realização de estudos observacionais em suas diversas modalidades podem proporcionar uma nova perspectiva no que tange os mecanismos fisiopatológicos subjacentes as duas doenças bem como a relação delas entre si. Tais estudos seriam de grande relevância e forneceriam informações relevantes contribuindo para o entendimento dos efeitos virais no organismo dos portadores de ELA. 


\section{Agradecimentos}

Deixamos nossos agradecimentos a todos os portadores de Esclerose Lateral Amiotrófica e também homenageamos os mais de 450.000 mil mortos por COVID-19 no Brasil.

\section{Referências}

Ajroud-Driss, S., \& Siddique, T. (2015). Sporadic and hereditary amyotrophic lateral sclerosis (ALS). Biochimica et Biophysica Acta (BBA) - Molecular Basis of Disease, 1852(4), 679-684. https://doi.org/10.1016/j.bbadis.2014.08.010

Bohmwald, K., Gálvez, N. M. S., Ríos, M., \& Kalergis, A. M. (2018). Neurologic Alterations Due to Respiratory Virus Infections. Frontiers in Cellular Neuroscience, 12(October), 1-15. https://doi.org/10.3389/fncel.2018.00386

Carod Artal, F. J. (2020). Complicaciones neurológicas por coronavirus y COVID-19. Revista de Neurología, 70(09), 311. https://doi.org/10.33588/rn.7009.2020179

Chia, R., Chiò, A., \& Traynor, B. J. (2018). Novel genes associated with amyotrophic lateral sclerosis: diagnostic and clinical implications. In The Lancet Neurology (Vol. 17, Issue 1, pp. 94-102). Lancet Publishing Group. https://doi.org/10.1016/S1474-4422(17)30401-5

Correia, A. O., Feitosa, P. W. G., Moreira, J. L. de S., Nogueira, S. Á. R., Fonseca, R. B., \& Nobre, M. E. P. (2020). Neurological manifestations of COVID19 and other coronaviruses: A systematic review. Neurology, Psychiatry and Brain Research, 37(January), 27-32. https://doi.org/10.1016/j.npbr.2020.05.008

Crepaldi, M. A., Schmidt, B., Noal, D. da S., Bolze, S. D. A., \& Gabarra, L. M. (2020). Terminalidade, morte e luto na pandemia de COVID-19: demandas psicológicas emergentes e implicações práticas. Estudos de Psicologia (Campinas), 37, 1-12. https://doi.org/10.1590/1982-0275202037e200090

Das, G., Mukherjee, N., \& Ghosh, S. (2020). Neurological Insights of COVID-19 Pandemic. ACS Chemical Neuroscience, 11(9), 1206-1209. https://doi.org/10.1021/acschemneuro.0c00201

De Felice, F. G., Tovar-Moll, F., Moll, J., Munoz, D. P., \& Ferreira, S. T. (2020). Severe Acute Respiratory Syndrome Coronavirus 2 (SARS-CoV-2) and the Central Nervous System. Trends in Neurosciences, 43(6), 355-357. https://doi.org/10.1016/j.tins.2020.04.004

Estrela, C. (2018). Metodologia Científica: Ciência, Ensino, Pesquisa (3a ed). https://books.google.com.br/books?id=67VIDwAAQBAJ\&lp g=PR1\&ots=87TD6S4sg5\&dq=Estrela\%2C C. (2018). Metodologia Científica\%3A Ciência\%2C Ensino\%2C Pesquisa. Editora Artes Médicas.\&lr\&hl=pt$\mathrm{BR} \& \mathrm{pg}=\mathrm{PR} 2 \# \mathrm{v}=$ onepage $\& \mathrm{q} \& \mathrm{f}=$ false

Filatov, A., Sharma, P., Hindi, F., \& Espinosa, P. S. (2020). Neurological Complications of Coronavirus Disease (COVID-19): Encephalopathy. Cureus, 12(3). https://doi.org/10.7759/cureus.7352

Gorbalenya, A. E., Baker, S. C., Baric, R. S., de Groot, R. J., Drosten, C., Gulyaeva, A. A., Haagmans, B. L., Lauber, C., Leontovich, A. M., Neuman, B. W., Penzar, D., Perlman, S., Poon, L. L. M., Samborskiy, D. V., Sidorov, I. A., Sola, I., \& Ziebuhr, J. (2020). The species Severe acute respiratory syndromerelated coronavirus: classifying 2019-nCoV and naming it SARS-CoV-2. Nature Microbiology, 5(4), 536-544. https://doi.org/10.1038/s41564-020-0695-Z

Guidon, A. C., \& Amato, A. A. (2020). COVID-19 and neuromuscular disorders. Neurology, 94(22), 959-969. https://doi.org/10.1212/WNL.0000000000009566

Hardiman, O., Al-Chalabi, A., Chio, A., Corr, E. M., Logroscino, G., Robberecht, W., Shaw, P. J., Simmons, Z., \& van den Berg, L. H. (2017). Amyotrophic lateral sclerosis. Nature Reviews Disease Primers, 3(1), 17071. https://doi.org/10.1038/nrdp.2017.71

Li, M.-Y., Li, L., Zhang, Y., \& Wang, X. (2020). Expression of the SARS-CoV-2 cell receptor gene ACE2 in a wide variety of human tissues. Infectious Diseases of Poverty, 9(1), 45. https://doi.org/10.1186/s40249-020-00662-X

Li, Y., Bai, W., \& Hashikawa, T. (2020). The neuroinvasive potential of SARS-CoV2 may play a role in the respiratory failure of COVID-19 patients. Journal of Medical Virology, 92(6), 552-555. https://doi.org/10.1002/jmv.25728

Lippi, A., Domingues, R., Setz, C., Outeiro, T. F., \& Krisko, A. (2020). SARS-CoV-2: At the Crossroad Between Aging and Neurodegeneration. Movement Disorders, 35(5), 716-720. https://doi.org/10.1002/mds.28084

Mehra, M. R., Desai, S. S., Ruschitzka, F., \& Patel, A. N. (2020). RETRACTED: Hydroxychloroquine or chloroquine with or without a macrolide for treatment of COVID-19: a multinational registry analysis. The Lancet. https://doi.org/10.1016/S0140-6736(20)31180-6

Meinhardt, J., Radke, J., Dittmayer, C., Franz, J., Thomas, C., Mothes, R., Laue, M., Schneider, J., Brünink, S., Greuel, S., Lehmann, M., Hassan, O., Aschman, T., Schumann, E., Chua, R. L., Conrad, C., Eils, R., Stenzel, W., Windgassen, M., \& Heppner, F. L. (2021). Olfactory transmucosal SARS-CoV-2 invasion as a port of central nervous system entry in individuals with COVID-19. Nature Neuroscience, 24(2), 168-175. https://doi.org/10.1038/s41593-020$00758-5$

Mejzini, R., Flynn, L. L., Pitout, I. L., Fletcher, S., Wilton, S. D., \& Akkari, P. A. (2019). ALS Genetics, Mechanisms, and Therapeutics: Where Are We Now? In Frontiers in Neuroscience (Vol. 13). https://doi.org/10.3389/fnins.2019.01310

Montalvan, V., Lee, J., Bueso, T., De Toledo, J., \& Rivas, K. (2020). Neurological manifestations of COVID-19 and other coronavirus infections: A systematic review. Clinical Neurology and Neurosurgery, 194(January), 105921. https://doi.org/10.1016/j.clineuro.2020.105921

Oh, J., \& Kim, J. A. (2017). Supportive care needs of patients with amyotrophic lateral sclerosis/motor neuron disease and their caregivers: A scoping review. Journal of Clinical Nursing, 26(23-24), 4129-4152. https://doi.org/10.1111/jocn.13945 
Research, Society and Development, v. 10, n. 7, e28310716441, 2021

(CC BY 4.0) | ISSN 2525-3409 | DOI: http://dx.doi.org/10.33448/rsd-v10i7.16441

Oskarsson, B., Gendron, T. F., \& Staff, N. P. (2018). Amyotrophic Lateral Sclerosis: An Update for 2018. In Mayo Clinic Proceedings (Vol. 93, Issue 11). https://doi.org/10.1016/j.mayocp.2018.04.007

Prado, L. de G. R., Vieira, É. L. M., Rocha, N. P., Prado, V. de G. R., Souza, L. C. de, \& Teixeira, A. L. (2018). Neuroinflamação Na Esclerose Lateral Amiotrófica. Revista Brasileira de Neurologia, 54(3), 22-27.

Serrano-Castro, P. J., Estivill-Torrús, G., Cabezudo-García, P., Reyes-Bueno, J. A., Ciano Petersen, N., Aguilar-Castillo, M. J., Suárez-Pérez, J., JiménezHernández, M. D., Moya-Molina, M. Á., Oliver-Martos, B., Arrabal-Gómez, C., \& Rodríguez de Fonseca, F. (2020). Impact of SARS-CoV-2 infection on neurodegenerative and neuropsychiatric diseases: A delayed pandemic? Neurología (English Edition), 35(4), 245-251. https://doi.org/10.1016/j.nrleng.2020.04.002

Taylor, J. P., Brown, R. H., \& Cleveland, D. W. (2016). Decoding ALS: from genes to mechanism. Nature, 539(7628), 197-206. https://doi.org/10.1038/nature20413

Tipton, P. W., \& Wszolek, Z. K. (2020). What can Parkinson's disease teach us about COVID-19? Neurologia i Neurochirurgia Polska, $368(2)$, m1091. https://doi.org/10.5603/PJNNS.a2020.0039

Toljan, K. (2020). Letter to the Editor Regarding the Viewpoint "Evidence of the COVID-19 Virus Targeting the CNS: Tissue Distribution, Host-Virus Interaction, and Proposed Neurotropic Mechanism" [Article-commentary]. ACS Chemical Neuroscience, 11(8), 1192-1194. https://doi.org/10.1021/acschemneuro.0c00174

van Doremalen, N., Bushmaker, T., Morris, D. H., Holbrook, M. G., Gamble, A., Williamson, B. N., Tamin, A., Harcourt, J. L., Thornburg, N. J., Gerber, S. I., Lloyd-Smith, J. O., de Wit, E., \& Munster, V. J. (2020). Aerosol and Surface Stability of SARS-CoV-2 as Compared with SARS-CoV-1. New England Journal of Medicine, 382(16), 1564-1567. https://doi.org/10.1056/NEJMc2004973

van Es, M. A., Hardiman, O., Chio, A., Al-Chalabi, A., Pasterkamp, R. J., Veldink, J. H., \& van den Berg, L. H. (2017). Amyotrophic lateral sclerosis. The Lancet, 390(10107), 2084-2098. https://doi.org/10.1016/S0140-6736(17)31287-4

Walhout, R., Verstraete, E., van den Heuvel, M. P., Veldink, J. H., \& van den Berg, L. H. (2018). Patterns of symptom development in patients with motor neuron disease. Amyotrophic Lateral Sclerosis and Frontotemporal Degeneration, 19(1-2), 21-28. https://doi.org/10.1080/21678421.2017.1386688

Wilson, M. P., \& Jack, A. S. (2020). Coronavirus disease 2019 (COVID-19) in neurology and neurosurgery: A scoping review of the early literature. Clinical Neurology and Neurosurgery, 193(April), 105866. https://doi.org/10.1016/j.clineuro.2020.105866

Yang, Y., Shang, W., \& Rao, X. (2020). Facing the COVID-19 outbreak: What should we know and what could we do? Journal of Medical Virology, 92(6), 536-537. https://doi.org/10.1002/jmv.25720

Yashavantha Rao, H. C., \& Jayabaskaran, C. (2020). The emergence of a novel coronavirus (SARS-CoV-2) disease and their neuroinvasive propensity may affect in COVID-19 patients. Journal of Medical Virology, 92(7), 786-790. https://doi.org/10.1002/jmv.25918

Zhou, Z., Kang, H., Li, S., \& Zhao, X. (2020). Understanding the neurotropic characteristics of SARS-CoV-2: from neurological manifestations of COVID-19 to potential neurotropic mechanisms. Journal of Neurology, 267(8), 2179-2184. https://doi.org/10.1007/s00415-020-09929-7

Zhu, N., Zhang, D., Wang, W., Li, X., Yang, B., Song, J., Zhao, X., Huang, B., Shi, W., Lu, R., Niu, P., Zhan, F., Ma, X., Wang, D., Xu, W., Wu, G., Gao, G. F., \& Tan, W. (2020). A Novel Coronavirus from Patients with Pneumonia in China, 2019. New England Journal of Medicine, 382(8), 727-733. https://doi.org/10.1056/nejmoa2001017 